\title{
Data-Driven Machine-Learning Quantifies Differences in the Voiding Initiation Network in Neurogenic Voiding Dysfunction in Women With Multiple Sclerosis
}

\author{
Christof Karmonik ${ }^{1,2}$, Timothy Boone ${ }^{1}$, Rose Khavari ${ }^{1}$ \\ ${ }^{1}$ Department of Urology, Houston Methodist Hospital, Houston, TX, USA \\ ${ }^{2}$ Translational Imaging Center, Houston Methodist Research Institute, Houston, TX, USA
}

\begin{abstract}
Purpose: To quantify the relative importance of brain regions responsible for reduced functional connectivity (FC) in their Voiding Initiation Network in female multiple sclerosis (MS) patients with neurogenic lower urinary tract dysfunction (NLUTD) and voiding dysfunction (VD). A data-driven machine-learning approach is utilized for quantification.

Methods: Twenty-seven ambulatory female patients with MS and NLUTD (group 1: voiders, $n=15$ and group 2: VD, $n=12$ ) participated in a functional magnetic resonance imaging (fMRI) voiding study. Brain activity was recorded by fMRI with simultaneous urodynamic testing. The Voiding Initiation Network was identified from averaged fMRI activation maps. Four machine-learning algorithms were employed to optimize the area under curve (AUC) of the receiver-operating characteristic curve. The optimal model was used to identify the relative importance of relevant brain regions.

Results: The Voiding Initiation Network exhibited stronger FC for voiders in frontal regions and stronger disassociation in cerebellar regions. Highest AUC values were obtained with 'random forests' (0.86) and 'partial least squares' algorithms (0.89). While brain regions with highest relative importance (>75\%) included superior, middle, inferior frontal and cingulate regions, relative importance was larger than $60 \%$ for 186 of the 227 brain regions of the Voiding Initiation Network, indicating a global effect.

Conclusions: Voiders and VD patients showed distinctly different FC in their Voiding Initiation Network. Machine-learning is able to identify brain centers contributing to these observed differences. Knowledge of these centers and their connectivity may allow phenotyping patients to centrally focused treatments such as cortical modulation.
\end{abstract}

Keywords: Neurogenic lower urinary tract dysfunction; Multiple sclerosis; Functional magnetic resonance imaging; Machine learning

- Research Ethics: This study was approved by the Institutional Review Board (IRB) of Houston Methodist Research Institute (IRB No. Pro00010110). Written informed consent was obtained from subjects participating in the study.

- Conflict of Interest: No potential conflict of interest relevant to this article was reported.

\footnotetext{
- HIGHLIGHTS

- Female MS patients with voiding dysfunction exhibit different FC patterns than those who void spontaneously.

- Machine learning algorithms are of advantage as they allow access to the complex nonlinearity of individual brain region FC for classification in voiding dysfunction.

- Differences in FC in voiding dysfunction are most pronounced in the left frontal brain and left cingulate.
}

Corresponding author: Christof Karmonik (D) https://orcid.org/0000-0002-6695-9535 Translational Imaging Center, Houston Methodist Research Institute, 6565 Fannin, Houston, TX, USA

E-mail: ckarmonik@houstonmethodist.org / Tel: +1-713-252-2661 /

Fax: +1-713-441-7295

Submitted: February 27, 2019 / Accepted after revision: May 27, 2019 


\section{INTRODUCTION}

As many as $90 \%$ of multiple sclerosis (MS) patients experience some sort of voiding dysfunction (VD) or incontinence [1]. The loss of neural conduction along axonal pathways that occurs in MS is caused by autoimmune-driven demyelination often accompanied by edema worsening the neurologic impairment. Depending on the affected regions in the central nervous system, symptoms vary greatly [2], generally characterized by neurogenic lower urinary tract dysfunction (NLUTD) [3] and may include difficulty of initiation or maintenance of voiding (VD).

Traditional clinical assessments such as urodynamic testing and urinary symptoms may not correlate with findings derived from standard clinical imaging [4]. Advanced neuroimaging methods, such as functional magnetic resonance imaging (fMRI) allow probing the involvement of regions in the brain and brainstem during micturition and have been recently successfully applied to female MS patients [5].

The demyelination of white matter tracts in MS affects functional connectivity (FC) in the brain [6]. A method to probe FC during micturition was recently presented [7]. In this approach, while undergoing a fMRI examination, subjects performed a voiding task during which blood oxygen level dependent (BOLD) signal time curves were recorded. Standard fMRI BOLD analysis provides a means to identify the brain network active during initiation of voiding. Strength of FC in this network is then derived from the degree of synchronicity of the BOLD signal time curves of corresponding voxels. The variation of FC in MS patients with VD may provide a more detailed picture of neural deficiency as it may directly relate to the underlying loss of anatomical connectivity while the activation/ deactivation of a particular brain region alone (as assessed by traditional fMRI BOLD analysis) may constitute a consequence of this impaired connectivity.

Distribution of white matter lesions in MS visible in standard clinical images vary individually. Large-scale diffusional changes in normal appearing white and gray matter have been reported thereby attributing a neurodegenerative component to MS [8].

While these findings may not allow generalization of FC disruptions, we hypothesize that differences in the Voiding Initiation Network in VD versus spontaneous voiders will be discernible.

Machine-learning algorithms furnish the technical means to assess the predictive power of FC strength of selected brain re- gions in the Voiding Initiation Network in order to distinguish these 2 patient groups. From the FC analysis, a complex interaction between different brain regions is obtained, which is represented by the correlation coefficient of the FMRI BOLD time courses. With machine-learning, this complexity can be handled efficiently, where all the connectivity information is used directly, in the training and classification approach. In addition, once trained, the machine-learning algorithms used here will allow providing a relative importance for each brain region.

Identifying these brain regions may lead to improvement of therapy, in particular of new approaches that aim to use brain stimulation techniques aimed at cortical modulation [9].

\section{MATERIALS AND METHODS}

\section{Subjects}

Functional MRI imaging data was used from a previously conducted prospective study approved by the Institutional Review Board [5]. Informed consent was obtained from subjects participating in the study. Twenty-seven ambulatory female patients were recruited with stable MS and NLUTD. Patients were separated into 2 groups: group 1 , voiders $(\mathrm{n}=15)$ and group 2, VD $(n=12)$. VD was defined as having postvoid residual of $\geq 40 \%$ of the maximum cystometric capacity or performing self-catheterization.

\section{fMRI Examination}

The detailed protocol of the fMRI examination has been reported previously [5]. Prior to the start of the fMRI examination (axial echo-planar imaging [EPI], TR $=3,000 \mathrm{~ms}, 4.0-\mathrm{mm}$ slice thickness, 3.38-mm in-plane resolution covering the entire brain, Philips Ingenia 3.0T, standard 12-channel head coil) and after the subject voided spontaneously or was catheterized, a double lumen 7Fr MRI-compatible bladder and rectal catheters were inserted to conduct urodynamic testing concurrent with the fMRI examination [10].

The bladder was gradually filled at $50 \mathrm{~mL} / \mathrm{min}$ with roomtemperature sterile saline until subjects signaled by hand movement a strong desire to void. After 30 seconds of instructed holding, the subjects were then given permission to initiate voiding. After voiding or attempt of voiding was completed, the cycle was repeated up to 4 times depending on the tolerance of the subject. Bladder was aspirated passively if patients we unable to void. Total duration of the fMRI examination was limit- 
ed to 45 minutes.

In addition to the fMRI EPI images, also a high-resolution T1-weighted image set was acquired (fast-field echo, sagittal direction, $0.7-\mathrm{mm}$ in-plane resolution) to serve as anatomical reference.

\section{fMRI Activation Maps}

Functional MRI image analysis was performed using the AFNI software suite (http://afni.nimh.nih.gov/afni) as described previously [5]. Functional and anatomical images were co-regis- tered, and motion correction was applied to the functional data. Significant activated voxels $(\mathrm{P}<0.05)$ were identified at the initiation of voiding under the generalized linear model (GLM). Group level analysis was performed by transforming data into Talairach space, and significantly activated voxels were identified using a Student t-test for both groups and an average BOLD activation map averaged over all subjects was created.

\section{Voiding Initiation Brain Network in MS}

The Voiding Initiation Network in MS was identified by the

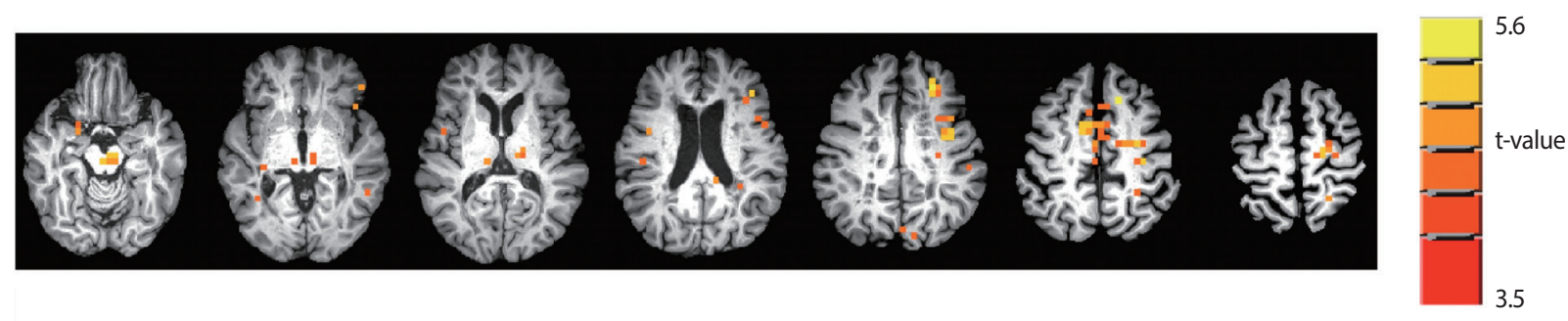

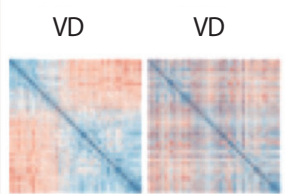

\#1

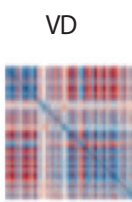

\#11

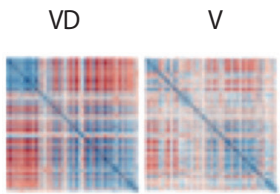

\#21
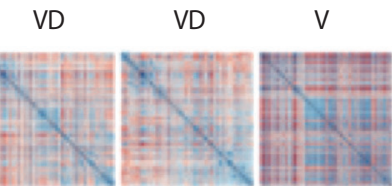

\#5

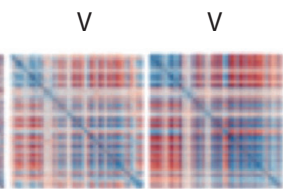

V

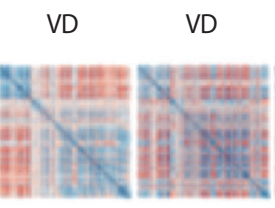

\#15
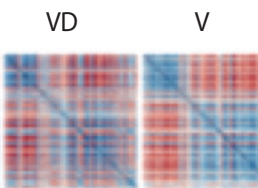

V

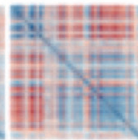

\#10

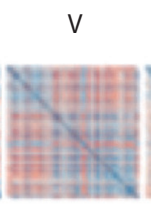

VD

V
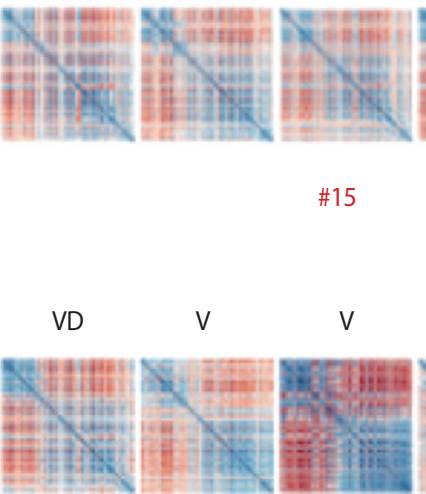

VD

V
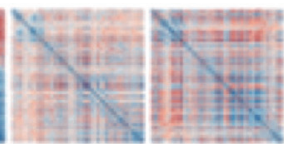

\#27

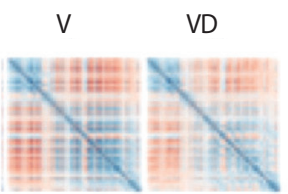

Average

Fig. 1. Upper panel: Voxels of the Voiding Initiation Network colored by functional magnetic resonance imaging blood oxygen level dependent activation overlaid onto brain anatomy in Talairach space. Lower panel: Individual and averaged functional connectivity (FC) patterns in the 27 multiple sclerosis patients participating in this study visualized by a color representation of the corresponding adjacency matrix (V, voider; VD, voiding dysfunction). In this presentation, vertices (i.e., voxels) of the Voiding Initiation Network are displayed vertically and horizontally. The intensity of the colored circle represents the strength of connection between each region (red: positive correlation, blue: negative correlation). As connections are undirected, the patterns are symmetric around the diagonal. While individual variation can be appreciated, VD globally exhibit weaker connections and larger variations. Consequently, while similar, the averaged FC pattern for VD is generally weaker and less coherent (lower right). 
most highly activated voxels $(t=3.5)$ in the averaged BOLD activation map and detailed in Supplementary material 1. This threshold was chosen as a compromise to include voxels in all pertinent brain regions, which exhibited activation in the averaged BOLD fMRI map $(\mathrm{P}<0.05)$, and at the same time to limit the number of predictor variables to render the data suitable for the machine-learning analysis. The resultant voxels $(n=227)$ defined the brain network of voiding initiation (Fig. 1).

For each subject, a FC graph network was created using the voxels of the Voiding Initiation Network as vertices. Edge weights were defined as the correlation coefficient of the BOLD signal time courses of the corresponding voxels centered around its mean and divided by their standard deviation (R-project, scale function).

Graph networks were visualized by their adjacency matrix (R-project, corrplot package) and by their connectivity pattern in Talairach anatomical space (Paraview, Kitware Inc., Clifton Park, NY, USA).

\section{Machine-Learning Analysis}

For each vertex of the individual Voiding Initiation Network, its strength (as the sum of its edge weights) was calculated. These values represented the predictor variables for the machinelearning analysis. The classifier variable was implemented as a binary vector $(0, \mathrm{VD} ; 1$, voiders).

The entire dataset was split into a training set (50\%) and a test set (50\%). Ten-fold repeated cross validation with 5 repeats was used to train the machine-learning algorithms (R-project, caret package).

The following 4 different machine-learning algorithms were employed and details are provided in Supplementary material 2. These 4 algorithms represent different concepts ranging from a simple linear approach (GLM) over a hybrid approach, where the original predictor variables are linearly combined (partial least squares), to highly nonlinear approach (neural network) and a classification approach (random forests). The use of these different concepts is motivated by the assumption that the structure of the data may be more suited for one of these approaches thereby yielding the best classification.

\section{Random forests}

An ensemble of decision trees is created where each tree uses a randomly selected subset of the predictors, which is smaller than the total number of predictors. Each tree is then used to generate a prediction from its subset and these predictions are averaged ('bagged') to give the final prediction.

\section{Neural networks}

Neural Networks are nonlinear regression techniques inspired by theories of neural interactions. The outcome is modeled by an intermediary set of unobserved variables ('hidden units'), which are linear combinations of the original predictors that are transformed by a nonlinear function. A linear combination of these hidden units is then related to the outcome.

\section{Generalized linear model}

The outcome is modeled by a linear combination of the original predictors.

\section{Partial least squares}

This algorithm iteratively seeks to find underlying, latent, relationships among the predictors that highly correlate with the response. Linear combinations of the original predictors are created maximizing their internal variation and their correlation with the response. By creating these linear combinations, the algorithm reduces the dimensionality of the predictor space, which is favorable if more predictor variables than response variables exist as is the case in this study. By combining the predictor variables, a more favorable ratio between these combined variables and the number of data available to train the algorithm is obtained with will improve the quality of the training.

Area under the curve (AUC) of the receiver-operating characteristic curve represented the cost function to determine the optimal algorithm.

\section{RESULTS}

\section{Voiding Initiation Network in MS Spatial extent}

The Voiding Initiation Network in MS was found to be widely distributed in the brain including areas such as the dorsal vagal motor nuclei, pontine storage and micturition center, periaqueductal gray (PAG), ventral tegmental area, substantia nigra, red nuclei, thalamus, cingulate and insula was well as cortical regions in the frontal, parietal, and mesial temporal lobes (Fig. 1, Supplementary material 1). Visualization in the anatomical Talairach space demonstrates a global connectivity pattern: for frontal brain regions the connection strength was mainly positive while cerebellar regions and selected limbic regions were more negative (Fig. 2). 

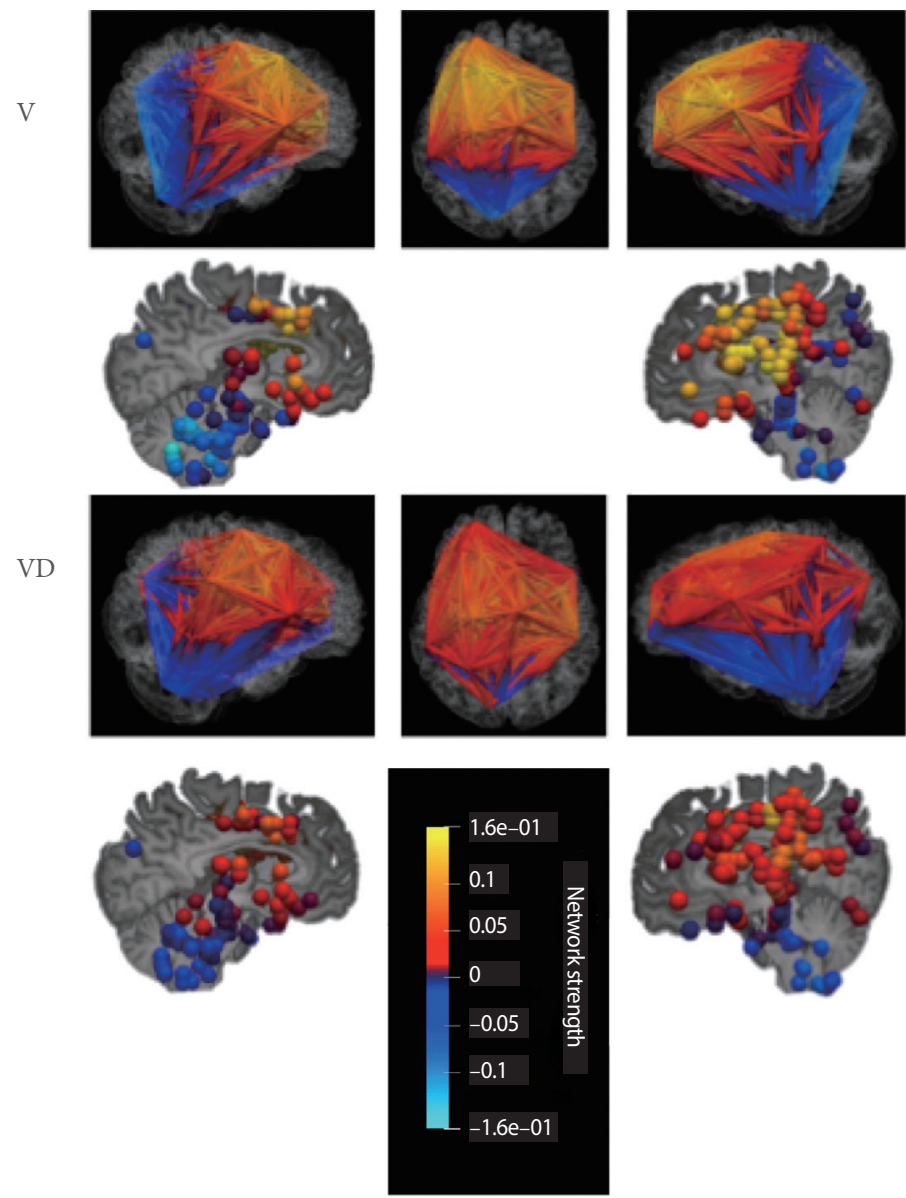
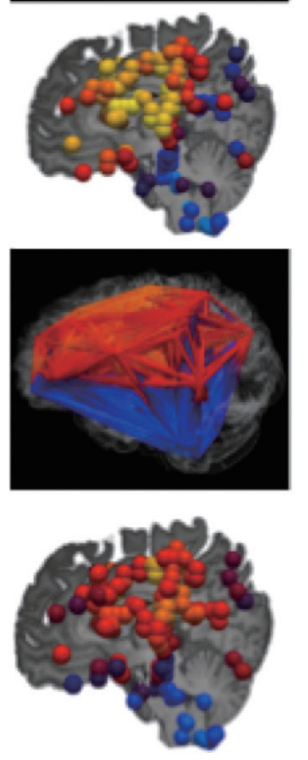

Connected

Left medial frontal gyrus

Left precentral gyrus

Left thalamus

Left inferior parietal lobule

Left precentral gyrus

Right insula

Left inferior frontal gyrus

Left middle frontal gyrus

Left middle frontal gyrus

Left cingulate gyrus

Right cingulate gyrus

Left cingulate gyrus

Left inferior frontal gyrus

Right thalamus

Left superior frontal gyrus

Left superior frontal gyrus

Right subcallosal gyrus

Left cingulate gyrus

Right thalamus

Left middle frontal gyrus

Periaqueductal gray

Left red nucleus

Left substantia nigra

Right culmen
Disassociated

Right inferior parietal lobule Left middle frontal gyrus

Left middle temporal gyrus

Right inferior frontal gyrus

Left inferior parietal lobule

Left superior frontal gyrus

Right hippocampus

Right red nucleus

Right uncus

Left fusiform gyrus

Fig. 2. Averaged functional connectivity patterns for voiders (Vs) and voiding dysfunction (VD) from Fig. 1 displayed in anatomical space. Color is based on graph network strength of the individual vertices (voxels). Lower maximum strength in the VD network, particular in the frontal lobes and the cerebellum, can be appreciated. On right, brain regions are listed in 2 columns. The first column (connected) identifies voxels of the Voiding Initiation Network (by location) where total connection strength is positive for VD and negative for $\mathrm{V}$. The second column (disassociation) lists those voxel of the Voiding Initiation Network (by location), where total connection strength is negative for VD and positive for V (see Supplementary material 1 for numerical values).

\section{Differences between voiders and voiding dysfunction}

While some variation existed between the individual networks, in general voiders exhibited stronger connections (Fig. 1). Similar to the individual networks, also the averaged network for voiders demonstrated stronger connections (Fig. 1).

Network components in the PAG, thalamus, substantia nigra, insula, subcallosal gyrus, hippocampus and in the frontal lobes were stronger connected for VD than voiders, whereas components in the temporal lobe hippocampus and fusiform gyrus were weaker connected for VD than voiders (Fig. 2, Supplementary material 1).

\section{Machine-Learning Analysis AUC values}

The 2 best-performing machine-learning algorithms were found to be partial least squares $(\mathrm{AUC}=0.89$ ) and random forests (AUC=0.86) (Fig. 3). AUC for the Neural Network algorithm was 0.71 and worst performance the GLM $(A U C=0.61)$ (Table 1).

\section{Performance on test data sets}

Evaluating the 2 best algorithms on the test data set yielded good values for accuracy ( 0.69 for partial least squares and 0.62 for random forests) (Table 2). 


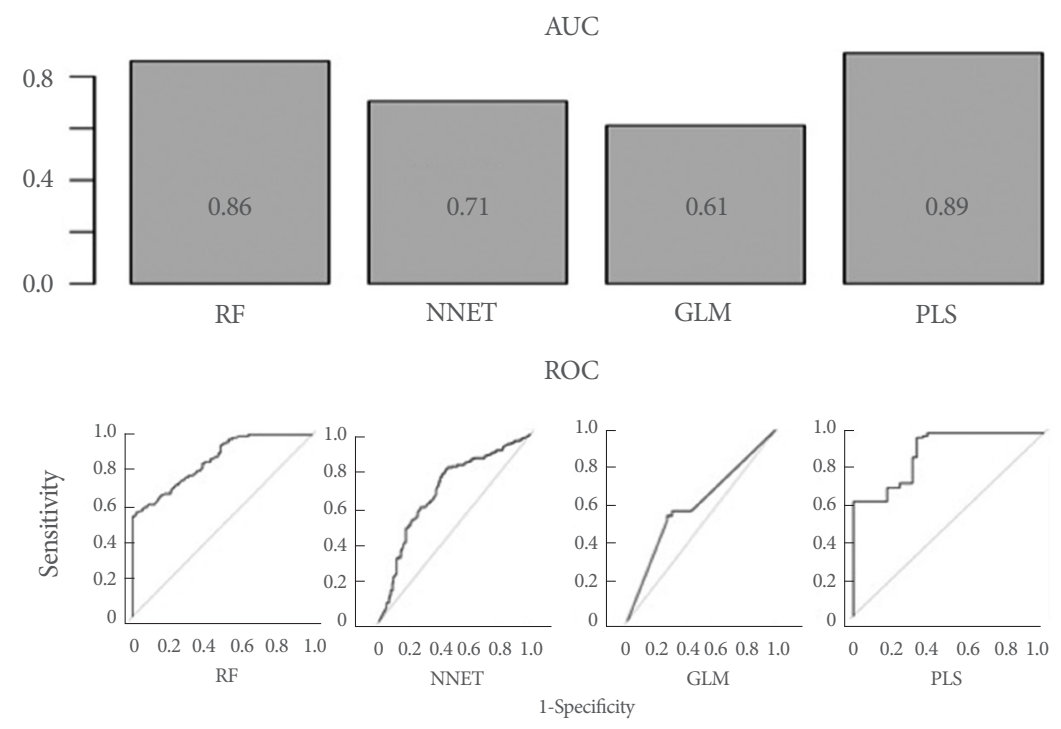

Fig. 3. Upper panel: Area under the curve (AUC) for all 4 machine-learning algorithms employed. AUC are provided. Lower panel: Receiver-operating characteristic curve (ROC) all 4 investigated machine-learning algorithms. Random forests (RF) and partial least squares (PLS) clearly outperform the other 2 algorithms. NNET, Neural Networks; GLM, generalized linear model.

Table 1. Area under the curve (AUC) values for the different models

\begin{tabular}{lc}
\hline Model & AUC \\
\hline RF & 0.86 \\
NNET & 0.71 \\
GLM & 0.61 \\
PLS & 0.89 \\
\hline
\end{tabular}

RF, random forests; NNET, Neural Networks; GLM, generalized linear model; PLS, partial least squares.

\section{Relative Importance of Brain Regions}

For the best-performing machine-learning model (partial least squares), brain regions with highest importance were located in the frontal lobes and the cingulate (Table 3, Fig. 4). Other regions of high importance included the precentral and parahippocampal gyrus as well as regions in the cerebellum (Fig. 4). However, no real 'cutoff' in the relative importance was observed, 25 regions showed importance higher than $70 \%$ and 186/227 voxels in the Voiding Initiation Network had a relative importance higher than $60 \%$ (Fig. 4, Table 3).

\section{DISCUSSION}

Our results demonstrate that patients with VD exhibit an altered functional connectome in their Voiding Initiation Net-
Table 2. Confusion matrices for the 2 models (\%)

\begin{tabular}{|c|c|c|c|c|}
\hline \multirow{3}{*}{ Prediction } & \multirow{2}{*}{\multicolumn{2}{|c|}{$\begin{array}{c}\text { Partial least square }{ }^{\mathrm{a})} \\
\text { Reference }\end{array}$}} & \multirow{2}{*}{\multicolumn{2}{|c|}{$\begin{array}{c}\text { Random forest }{ }^{\mathrm{b})} \\
\text { Reference }\end{array}$}} \\
\hline & & & & \\
\hline & VD & $\mathrm{V}$ & VD & $\mathrm{V}$ \\
\hline VD & 31 & 15 & 31 & 23 \\
\hline V & 15 & 39 & 15 & 31 \\
\hline
\end{tabular}

$\mathrm{V}$, voider; $\mathrm{VD}$, voiding dysfunction.

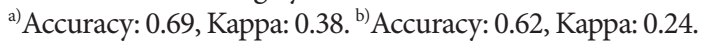

work where largest variations relative to voiders are found in the frontal lobes and the cingulate.

Earlier neuroimaging studies have identified brain regions directly involved in initiating or continuing voiding in healthy individuals. These regions include: precentral gyrus, supplementary motor area, dorsolateral prefrontal lobe, inferior frontal gyrus, cingulate gyrus, insula, hypothalamus, PAG, and pons (PMC) [9-12], specifically the cingulate region is highly activated at full capacity and with strong desire to void (immediately prior to voiding) in healthy and MS patients [10-13]. Frontal and prefrontal regions of the brain combine all the input from cingulate, insula and hypothalamus and further the decision to void is made when it is the right time, the right place and with appropriate bladder volume [14,15].

Due to the diffuse, multifocal disease involvement of the 
Table 3. Relative importance (PLS) of first 30 most important voxels of the Voiding Initiation Network that differentiate between voiders and voiding dysfunction (Fig. 4)

\begin{tabular}{|c|c|c|c|c|}
\hline Region & $\mathrm{R}(\mathrm{mm})$ & $\mathrm{A}(\mathrm{mm})$ & $\mathrm{I}(\mathrm{mm})$ & PLS \\
\hline Left medial frontal gyrus & 2.5 & -22.5 & 47.5 & 100.0 \\
\hline Left cingulate gyrus & 2.5 & -2.5 & 47.5 & 98.4 \\
\hline Left middle frontal gyrus & 42.5 & -12.5 & 32.5 & 97.3 \\
\hline Left inferior frontal gyrus & 52.5 & -7.5 & 17.5 & 94.9 \\
\hline Left inferior frontal gyrus & 52.5 & -7.5 & 22.5 & 94.5 \\
\hline Left medial frontal gyrus & 2.5 & -7.5 & 47.5 & 93.0 \\
\hline Left middle frontal gyrus & 47.5 & -12.5 & 27.5 & 86.9 \\
\hline Left medial frontal gyrus & 7.5 & -7.5 & 47.5 & 86.9 \\
\hline Left cingulate gyrus & 7.5 & -22.5 & 42.5 & 86.0 \\
\hline Left middle frontal gyrus & 27.5 & 7.5 & 57.5 & 85.2 \\
\hline Left fusiform gyrus & 42.5 & 32.5 & -22.5 & 84.6 \\
\hline Left medial frontal gyrus & 7.5 & -22.5 & 47.5 & 83.3 \\
\hline Left cingulate gyrus & 2.5 & -7.5 & 42.5 & 80.1 \\
\hline Left inferior frontal gyrus & 42.5 & -2.5 & 32.5 & 79.2 \\
\hline Left inferior parietal lobule & 32.5 & 27.5 & 27.5 & 77.6 \\
\hline Dorsal vagal motor nuclei & 2.5 & 37.5 & -42.5 & 74.7 \\
\hline Left cingulate gyrus & 12.5 & 32.5 & 27.5 & 74.6 \\
\hline Right cingulate gyrus & -7.5 & -17.5 & 42.5 & 74.3 \\
\hline Right inferior semi-lunar lobule & -12.5 & 62.5 & -37.5 & 74.2 \\
\hline Dorsal vagal motor nuclei & -2.5 & 32.5 & -47.5 & 73.8 \\
\hline Left superior frontal gyrus & 32.5 & -37.5 & 32.5 & 73.8 \\
\hline Left cingulate gyrus & 17.5 & 37.5 & 27.5 & 73.4 \\
\hline Left superior frontal gyrus & 27.5 & -37.5 & 32.5 & 73.3 \\
\hline Left precentral gyrus & 22.5 & 12.5 & 52.5 & 71.0 \\
\hline Left precentral gyrus & 27.5 & 12.5 & 52.5 & 68.7 \\
\hline Right uvula & -7.5 & 62.5 & -32.5 & 67.7 \\
\hline Left inferior frontal gyrus & 47.5 & -12.5 & 22.5 & 67.5 \\
\hline Left substantia nigra & 7.5 & 17.5 & -7.5 & 67.2 \\
\hline Left cingulate gyrus & 12.5 & 37.5 & 22.5 & 65.6 \\
\hline
\end{tabular}

$\mathrm{R}$, right; A, anterior; I, inferior; PLS, partial least squares.

brain in MS, anatomical connectivity and therefore $\mathrm{FC}$ is thought to be impaired on a global level [8]. Individual differences in the urinary symptom severity and impact on quality of life exist. This clinical observation is in concordance with our findings of global impairment of the Voiding Initiation Networks for VD patients compared to voiders. It is also in agreement with a reduction in similarity of the global FC patterns with disease progression reported recently [7].

Our approach complements standard clinical testing and es- tablished traditional fMRI BOLD techniques, which focuses solely on activation of brain regions. By being able to characterize the global FC connectome, our method allows monitoring FC changes with disease progression as well as therapeutic interventions. Of the latter, noninvasive brain stimulation has gained interest recently. It constitutes a promising new intervention in MS that has already yielded promising results for motor and cognitive function in combination with appropriate training exercises [9]. Identifying potential brain regions as tar- 

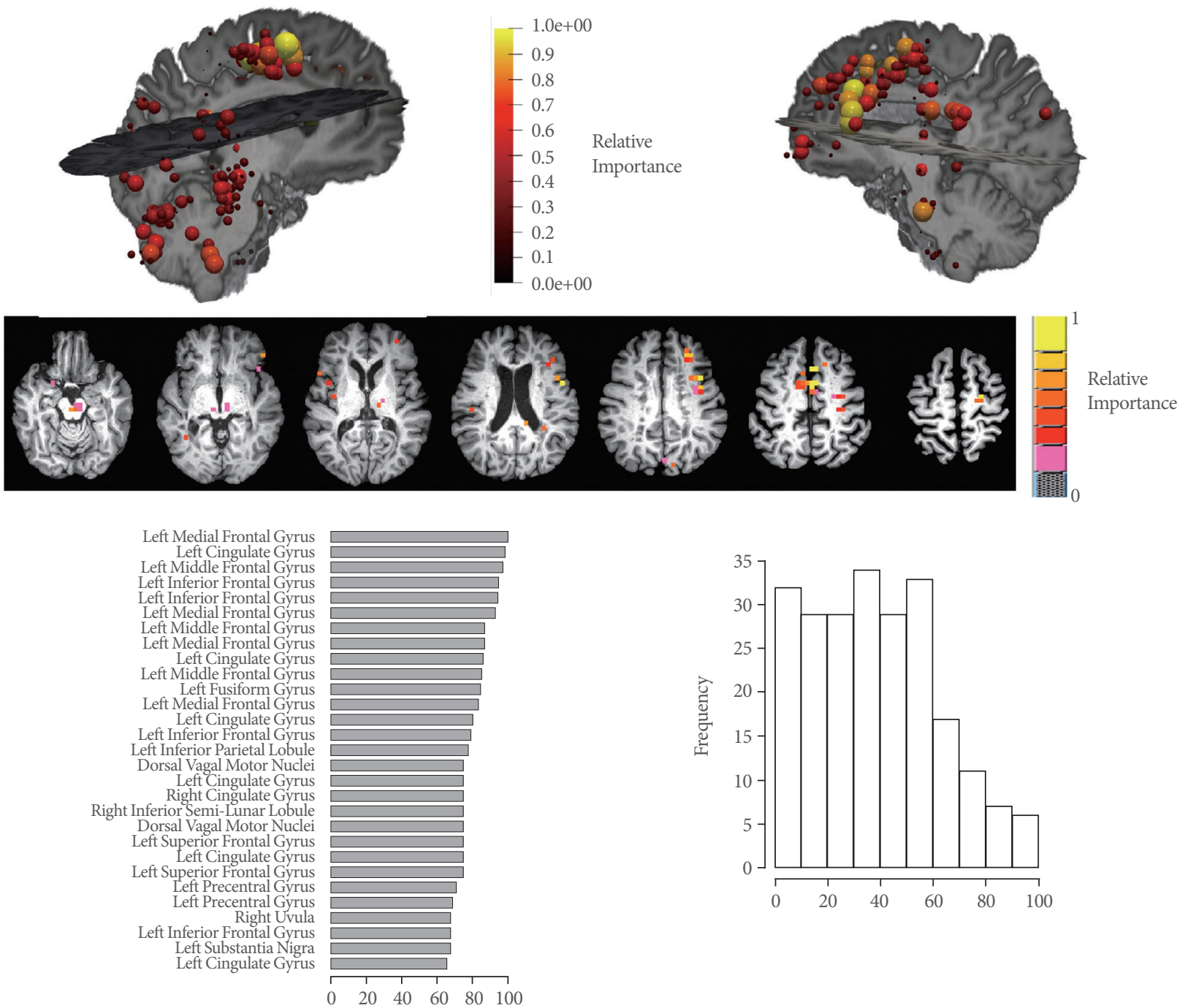

Fig. 4. Upper panel: Relative importance differentiating functional connectivity (FC) in voiders and voiding dysfunction for vertices belonging to the Voiding Initiation Network displayed in anatomical space. Individual vertices corresponding to voxels in the brain are display in pseudo-color and size according the importance. Highest importance is found in the frontal lobes and cerebellum, but is generally high for all voxels. Middle panel: Voxel from top panel displayed in axial orientation in Talairach space. Lower panel: On left, the 30 top vertices (by location) of the Voiding Initiation Network with highest relative importance (in percent) in each hemisphere (coordinates are provided in Table 3). On right, histogram over all 227 voxels demonstrating the high relative importance for a large number of these voxels supporting the conclusion that FC is affected globally.

get for stimulation (e.g., frontal regions) and monitoring global FC changes during and after stimulation may be essential for optimizing stimulation techniques.

Machine-learning algorithms have found recent applications in correlating clinical neuropsychological findings with brain connectivity measures. Examples include brain-computer interface stroke rehabilitation $[11,12]$, discrimination of schizophrenia [13] and mild traumatic brain injury $[14,15]$. In con- trast to these approaches, in our method, no assumptions are made about significance of connections by applying a threshold. Instead, the entire functional connectome of the Voiding Initiation Network is used as input into the machine-learning algorithms thereby improving the data-driven nature of the paradigm.

The number of subjects $(\mathrm{n}=27)$ for a machine-learning approach is somewhat limited, in particular, if compared with the 
number of predictor variables (i.e., number of brain voxels of the Voiding Initiation Network). The use of advanced algorithms, such as partial least squares or neural networks, where the original predictor variables are combined, thereby reducing the dimensionality of the predictor space, creates a more favorable ratio between this number of variables and the number of available data for training the algorithm. This in turn will help to arrive at a better trained algorithm. Such an approach therefore successfully addresses the limited number of clinical datasets, as they often exist in a single center clinical study. Alternatively, the machine-learning algorithm may be trained by combining data from several clinical sites. Once trained, it can then be applied to any single center study.

Two algorithms, random forests and partial least squares performed best according to their AUC values (0.86 and 0.89, respectively). Therefore, we conclude that these 2 algorithms are best suited for the classification problem in this study.

Understanding supraspinal centers and their role in initiating or modulating voiding is currently still limited in patients with neurogenic VD. By combining new concepts of neuroimaging with powerful machine-learning algorithms, we increase the knowledge of these processes and aid in phenotyping patients for novel treatment approaches such as brain stimulation.

In conclusion, machine-learning is capable of quantifying FC differences in MS patients with NLUTD and VD. The functional connectome of the Voiding Initiation Network is less coherent in this patient group. Global differences were found with focal points in the frontal lobes and the cingulate.

\section{SUPPLEMENTARY MATERIALS}

Supplementary materials 1-2 can be found via https://doi. org/10.5213/inj.1938058.029.

\section{AUTHOR CONTRIBUTION STATEMENT}

- Full access to all the data in the study and takes responsibility for the integrity of the data and the accuracy of the data analysis: $R K$

- Study concept and design: $R K$

- Acquisition of data: $C K$

- Analysis and interpretation of data: $C K$

- Drafting of the manuscript: $C K$

- Critical revision of the manuscript for important intellectual content: $R K, T B$
- Statistical analysis: $C K$

- Obtained funding: $R K$

- Administrative, technical, or material support: $C K$

-Study supervision: $R K$

\section{REFERENCES}

1. Corcos J. A urological challenge: voiding dysfunction in multiple sclerosis. Can Urol Assoc J 2013;7(9-10 Suppl 4):S181-2.

2. Wintner A, Kim MM, Bechis SK, Kreydin EI. Voiding dysfunction in multiple sclerosis. Semin Neurol 2016;36:34-40.

3. Stöhrer M, Blok B, Castro-Diaz D, Chartier-Kastler E, Del Popolo G, Kramer G, et al. EAU guidelines on neurogenic lower urinary tract dysfunction. Eur Urol 2009;56:81-8.

4. Kim YH, Goodman C, Omessi E, Rivera V, Kattan MW, Boone TB. The correlation of urodynamic findings with cranial magnetic resonance imaging findings in multiple sclerosis. J Urol 1998;159:972-6.

5. Khavari R, Elias SN, Pande R, Wu KM, Boone TB, Karmonik C. et al. Higher neural correlates in patients with multiple sclerosis and neurogenic overactive bladder following treatment with intradetrusor injection of onabotulinumtoxinA. J Urol 2019;201:135-40.

6. Tahedl M, Levine SM, Greenlee MW, Weissert R, Schwarzbach JV. Functional connectivity in multiple sclerosis: recent findings and future directions. Front Neurol 2018;9:828.

7. Khavari R, Elias SN, Boone T, Karmonik C. Similarity of functional connectivity patterns in patients with multiple sclerosis who void spontaneously versus patients with voiding dysfunction. Neurourol Urodyn 2019;38:239-47.

8. Hasan KM, Walimuni IS, Abid H, Datta S, Wolinsky JS, Narayana PA. Human brain atlas-based multimodal MRI analysis of volumetry, diffusimetry, relaxometry and lesion distribution in multiple sclerosis patients and healthy adult controls: implications for understanding the pathogenesis of multiple sclerosis and consolidation of quantitative MRI results in MS. J Neurol Sci 2012;313:99109.

9. Blok BF, Sturms LM, Holstege G. Brain activation during micturition in women. Brain 1998;121(Pt 11):2033-42.

10. Blok BF, Willemsen AT, Holstege G. A PET study on brain control of micturition in humans. Brain 1997;120(Pt 1):111-21.

11. Shy M, Fung S, Boone TB, Karmonik C, Fletcher SG, Khavari R. Functional magnetic resonance imaging during urodynamic testing identifies brain structures initiating micturition. J Urol 2014; 192:1149-54.

12. Nour S, Svarer C, Kristensen JK, Paulson OB, Law I. Cerebral activation during micturition in normal men. Brain 2000;123(Pt 4): 
781-9.

13. Khavari R, Karmonik C, Shy M, Fletcher S, Boone T. Functional magnetic resonance imaging with concurrent urodynamic testing identifies brain structures involved in micturition cycle in patients with multiple sclerosis. J Urol 2017;197:438-44.

14. Kuhtz-Buschbeck JP, Gilster R, van der Horst C, Hamann M, Wolff
S, Jansen O. Control of bladder sensations: an fMRI study of brain activity and effective connectivity. Neuroimage 2009;47:18-27.

15. Kuhtz-Buschbeck JP, van der Horst C, Pott C, Wolff S, Nabavi A, Jansen $\mathrm{O}$, et al. Cortical representation of the urge to void: a functional magnetic resonance imaging study. J Urol 2005;174(4 Pt 1):1477-81. 